\title{
Research Article Operating Room Scheduling in Teaching Hospitals
}

\author{
Somayeh Ghazalbash ${ }^{1}$ Mohammad Mehdi Sepehri, ${ }^{1,2}$ \\ Pejman Shadpour, ${ }^{2}$ and Arezoo Atighehchian ${ }^{1}$
}

${ }^{1}$ Department of Industrial Engineering, Tarbiat Modares University, Tehran 14117, Iran

${ }^{2}$ Hospital Management Research Center, Tehran University of Medical Sciences, Tehran 19697, Iran

Correspondence should be addressed to Mohammad Mehdi Sepehri, mehdi.sepehri@gmail.com

Received 7 October 2011; Accepted 3 December 2011

Academic Editor: Ching-Jong Liao

Copyright (C) 2012 Somayeh Ghazalbash et al. This is an open access article distributed under the Creative Commons Attribution License, which permits unrestricted use, distribution, and reproduction in any medium, provided the original work is properly cited.

\begin{abstract}
Operating room scheduling is an important operational problem in most hospitals. In this paper, a novel mixed integer programming (MIP) model is presented for minimizing Cmax and operating room idle times in hospitals. Using this model, we can determine the allocation of resources including operating rooms, surgeons, and assistant surgeons to surgeries, moreover the sequence of surgeries within operating rooms and the start time of them. The main features of the model will include the chronologic curriculum plan for training residents and the real-life constraints to be observed in teaching hospitals. The proposed model is evaluated against some real-life problems, by comparing the schedule obtained from the model and the one currently developed by the hospital staff. Numerical results indicate the efficiency of the proposed model compared to the real-life hospital scheduling, and the gap evaluations for the instances show that the results are generally satisfactory.
\end{abstract}

\section{Introduction}

Health care expenditures comprise a meaningful portion of the Gross Domestic Product in both developed and developing countries. Expenditure on healthcare in the UK as a percentage of the UK Gross Domestic Product (GDP) was estimated to be $8.4 \%$ in 2007, from which the public share was $69 \%$ [1]. Also, according to the statics released by the WHO (World Health Organization), health care expenditures in 2007 in Iran as a developing country were estimated to be about $6.4 \%$ of its GDP, and the portion covered by the government was about $46.8 \%$. This fact makes health systems an important research field for industrial engineering and operations research to improve their operational efficiency.

Operating rooms are simultaneously the largest cost centers and the greatest source of revenues for most hospitals. OR planning and scheduling is a key tool which can be useful to improve the productivity level of ORs and the related departments. 
Basically, there are three OR scheduling strategies commonly employed:

(1) blocked scheduling strategy,

(2) open scheduling strategy, and

(3) modified scheduling strategy.

Under a blocked scheduling strategy, individual surgeons or surgical groups are assigned times in a particular OR in a periodic (typically weekly or monthly) schedule. The planning within the framework of a blocked strategy consists of three stages. In the first stage, the OR capacity is divided among the surgeons, surgical groups, or departments on a strategic level. Then, a cyclic timetable called "Master Surgical Schedule" is constructed that defines the number and type of operating rooms available, the hours that ORs will be open, and the surgical groups or surgeon sessions for each OR [2]. The last stage which may be called "surgery process scheduling" splits into two subproblems called "advance scheduling" and "allocation scheduling" [3]. The first subproblem at a tactical level (one weak to one month) solves a planning phase by assigning an operating date to each patient over the time horizon. The second subproblem solves a scheduling phase which determines the sequence and resource allocation of cases in a given day $[4,5]$.

In the open strategy, the hospital does not hold operation rooms specific to a single surgeon although there is sometimes a weekly schedule for each surgeon. In this strategy, the intention is to accommodate all patients. The surgeons submit cases up until the day of the surgery, and all the cases are scheduled in ORs.

The modified strategy is similar to the blocked one except that certain slots in the master surgical schedule are left open for flexibility. In fact, this strategy is a mix of open and blocked strategies.

In this paper, we investigate the deterministic daily scheduling of surgical cases in teaching hospitals using the open scheduling strategy. A novel MIP model is presented with the objective of minimizing the total idle times of operating rooms and $\mathrm{Cmax}$ which represent completion time of the last patient's surgery. Using this model, we endeavor to allocate the required resources including operating rooms, surgeons, and assistant surgeons to surgeries and also determine the sequence of surgeries within operating rooms and the start time of each surgery. The major features of the model include the chronologic curriculum plan for training residents and the real-life constraints in teaching hospitals. We maintain that the problem defined here with its specific characteristics is not reported in the literature and that it finds real applications in teaching hospitals.

The rest of the paper is organized as follows. A brief review of previous work will first be presented in Section 2. Section 3 will provide a definition of the problem. The MIP model will be presented in Section 4. Section 5 is devoted to solving approach. Numerical results will be reported in Section 6. Conclusions will be drawn and presented in Section 7.

\section{A Literature Review}

Over the past 60 years, a large body of literature has evolved on the management of operating theaters. Magerlein and Martin [3] reviewed the literature on surgical demand scheduling and, as previously mentioned, focused on "surgery process scheduling" to differentiate between advance and allocation scheduling. Blake and Carter [6] extended this classification and added the domain of external resource scheduling which is defined as the process of identifying and reserving all resources external to the surgical suite necessary 
to ensure appropriate care for a patient before and after an instance of surgery. They further divided each domain into strategic, administrative, and operational levels [7]. Many authors (Magerlein and Martin, 1987; Kennedy [8]) classified the literature based on solution techniques [4], but Cardoen et al. [7] proposed a detailed classification based on 7 areas that are related to either the problem setting (e.g., performance measures or patient classes) or the technical features (e.g., solution technique or uncertainty incorporation) and analyzed the contributions in these areas.

Deterministic and stochastic mathematical programming models, queuing models, simulation models, and heuristic approaches have all been widely used to investigate OR scheduling. In this paper, we will briefly focus on the studies that have a direct bearing on surgical process scheduling and will consider the deterministic programming models used for OR scheduling. A more comprehensive review can be found in [7].

Ogulata and Erol [9] modeled the hierarchical multiple criteria mathematical programming to assign surgeon groups and dates to patients. A goal programming has been proposed to solve this weekly programming. Vissers et al. [10] presented an MIP model for developing a master operating theater schedule for thoracic surgery. Chaabane et al. [2] introduced two planning methods to compare open and blocked strategies. A weekly surgical case planning has been introduced to assign OR, operation day, and start time to patients in the open strategy, and an MSS has been defined for the blocked strategy. Jebali et al. [11] split a surgical case scheduling into 2 stages. First, the surgical cases were assigned to OR and then sequenced based on two sequencing strategies. Testi et al. [12] used the blocked strategy to develop a three-phase hierarchical approach for the weekly planning of surgical cases. They also applied a simulation method to analyze different rules of surgical case sequencing such as LWT, LPT, and SPT. Fei et al. [13] focused on the planning stage and assigned a set of surgical cases to OR with the objective of minimizing total operating cost. They proposed a branch and price algorithm to solve this problem for the exact solution. This model is similar to a parallel machine scheduling problem. Pham and Klinkert [4] Proposed a novel extension of the job shop scheduling problem called multimode blocking job shop to construct a surgical case scheduling for elective and add-on cases. Riis and Burke [14] use a discrete time model for surgery scheduling problem. Their model is similar to the one-step job shop scheduling problem with multiple identical machines, and they present a metaheuristic local-search-based algorithm for solving it. Cardoen et al. [15] investigated the sequencing of outpatients in an ASC (Ambulatory Surgery Center) in which balancing the use of recovery beds and medical precaution constraints such as additional sterilizing of OR after surgery of infected patients and sterilizing of medical instruments were taken into account. A branch and price methodology was used to solve this NP-hard optimization problem. Fei et al. [16] employed the open strategy to design a weekly surgery schedule. They solved the problem in two phases to give the surgery date for each patient and to determine the sequence of operations in each operating room on each day. Roland et al. [5] introduced a RCPSP (Resource Constraint Project Scheduling Problem) to generate scheduling and planning or surgical cases while focusing as much attention on human resources as on economic factors. They proposed a heuristic solution procedure based on genetic algorithm for solving this problem.

Hanset et al. [17] studied a daily scheduling problem, including material and human constraints. Their problem is modeled and solved via a constraint programming approach. Hanset et al. [18] compared their constraint programming model, [17], to a mixed integer programming model previously published by Roland et al. [5] that was adapted for the need of their study. 
In almost all the studies cited above, the surgeon for each case was known in advance. In this paper, the daily scheduling of surgical cases in teaching hospitals is investigated in which the training operations must be performed by postgraduate alumni, called "residents" or "fellows," under the supervision of a relevant attending surgeon while they are also qualified to do certain operations independently.

Chronologic curriculum plan for training residents is addressed in resident scheduling problem (RSP) repeatedly, which is different from operating room scheduling problem. This problem involves assigning residents to day and night shifts over a given planning horizon subject to numerous working regulations and staffing requirements [19]. For example, Topaloglu and Ozkarahan [19] developed a mixed-integer programming model for scheduling residents' duty hours considering the on-call night, dayoff, rest period, and total work-hour ACGME (Accreditation Council for Graduate Medical Education) regulations as well as the demand coverage requirements of the residency program. Sherali et al. [20] addressed the resident scheduling problem (RSP) at hospitals concerned with prescribed work nights for residents while considered departmental staffing and skill requirements as well as residents' preferences.

Dexter et al. [21] developed a methodology to determine rotations consisting of combinations of specialties to be paired for purposes of trainee scheduling to reduce the incidence of daily assignments off rotation.

To the best of our knowledge among the body of work on OR planning and scheduling that has appeared in the literature, no report is available in the literature on operating room scheduling in teaching hospitals that takes into account real constraints such as the chronologic curriculum plan for training residents. Constraints related with allocation of operations based on different seniority levels of residents and fellowships and providing a good balance between total hours of operations that performed by them than the average in a month. Also most of these papers assume that surgeon of each patient is already known. So it seems, assigning surgeons, residents, and assistant surgeons to each instance of surgery in literature of OR planning and scheduling is new.

\section{Problem Definition}

The process of surgical scheduling consists of two steps. The first concerns a weekly plan to assign a specific date to each patient waiting for surgery while the second involves sequencing and scheduling surgical cases on a given date. In this paper, we consider a surgical case scheduling problem (the second step) in a teaching hospital. In such hospitals, a list is prepared of the patients waiting for operation on the following day. Both inpatients and outpatients are scheduled each day by the OR head nurse or manager who sequences the surgeries and assigns a surgeon, an operating room, and a start time to each surgery.

Patients are prioritized and sequenced by the head nurse based on both resource availability (the eligible surgeon, the required equipments and aids, and the appropriate OR) and patient (child or old) priority.

The training operations must be performed by postgraduate alumni ("residents" and "fellows") under the supervision of relevant attending surgeons. The residents and fellows are usually grouped according to their seniority levels, and each of them is qualified to do certain operations based on their experience and qualifications acquired in the course of their curriculum plan. This plan determines the type of operations that can be performed by each resident or fellow group during each period of their education. 
Certain operations also require an assistant surgeon. The distribution of surgeries among surgeon groups, residents, and fellows should be based on equal opportunity for all to acquire experience. The nontraining operations are performed by public surgeons.

Some surgeries require special equipments which are available in particular ORs. For each operation, a group of medical staff members collectively called the surgery aid group in this paper, consisting of a scrub nurse, a circular nurse, and an anesthetic technician, is required. Also several medical instruments are needed during the surgery. After each surgery, instruments possibly need to be sterilized for some periods and, hence, are not available for subsequent surgeries.

We should also deal with the likelihood of infection spread. Therefore, special sanitary procedures must be executed to avoid the transfer of infection from patient to patient. In particular, the operating room needs additional cleaning after operation on an infected patient.

In the next section, the objectives and constraints of the problem are identified in a mathematical formulation.

\section{Mathematical Formulation of the Problem}

This section describes an MIP model for determining the daily surgical scheduling of elective patients (inpatient and outpatient) that calls "HORS" (hospital operating room scheduling).

\subsection{Notations}

The following notations are used in this paper.

(i) Indexes

$$
\begin{aligned}
& i \text { : Index for surgery. } \\
& r \text { : Index for operating room. } \\
& s, s^{\prime} \text { : Index for surgeon and assistant surgeon. } \\
& p \text { : Index for time slot (30-minute units). } \\
& k \text { : Index for medical instrument type. } \\
& l \text { : Index for group number. }
\end{aligned}
$$

(ii) Inputs

$\operatorname{AtN}(i, s)$ : If operation $i$ should be performed under the supervision of an attending surgeon $s$, it take 1 ; otherwise, 0 .

$\mathrm{d}(i)$ : Duration of operation $i$.

FAS $(i, s)$ : If surgeon $s$ can be the assistant for operation $i$, it takes 1 ; otherwise, 0 .

$\operatorname{FR}(i, r)$ : If surgery $i$ can be performed in operating room $r$, it takes 1 ; otherwise, 0 .

$\mathrm{FS}(i, s)$ : If surgery $i$ can be performed by surgeon $s$ it takes 1 ; otherwise, 0 .

Infect $(i)$ : If the patient of surgery $i$ is infected, it takes 1 ; otherwise, 0.

$\operatorname{Ins}(i, k)$ : If surgery $i$ needs instrument $k$, it takes 1 ; otherwise, 0 . 
IP0(k): The initial inventory of instrument sets of type $k$.

Nat $(i)$ : The number of required attending surgeons for the supervision of operation $i$.

IsNA $(i)$ : If operation $i$ needs an assistant surgeon, it takes 1 ; otherwise, 0 .

$\mathrm{GN}(s, l)$ : If surgeon $s$ to be of group number $l$, it takes 1 ; otherwise, 0 .

priority $(i)$ : If patient $i$ be a priority patient, it takes 1 ; otherwise, 0 .

$\operatorname{SAV}(s, p)$ : If surgeon $s$ is available on block $p$, it takes 1 ; otherwise, 0 .

$\mathrm{SGC}(s)$ : Surgeon group type: for residents it takes 1 , for fellows it takes 2 , and for attending surgeons it takes 3.

$\operatorname{TecNo}(p)$ : Number of technicians available on block $p$.

$\mathrm{TS}(k)$ : The duration of sterilization of instrument $k$.

Kclean: The duration of additional cleaning of OR after the surgery of an infected patient.

mean $(l)$ : Average total operation time assigned to group number $l$ in one month.

$\operatorname{dev}(l)$ : Allowable deviation from mean $(l)$.

C0: Last time allowed for the operating room doing surgery.

P0: Maximum time allowed to start of priority surgery.

(iii) Decision Variables

$X_{i p s r}$ : If surgery $i$ starts in period $p$ by surgeon $s$ in room $r$, it takes 1 ; otherwise, 0 .

$Y_{i p s r}$ : If surgery $i$ occupies period $p$ in room $r$ by surgeon $s$, it takes 1 ; otherwise, 0 .

$A_{\text {isp }}$ : If surgery $i$ is assigned to assistant surgeon $s$ in period $p$, it takes 1 ; otherwise, 0 .

$\mathrm{AP}_{i s p}$ : If surgery $i$ occupies period $p$ by assistant surgeon $s$, it takes 1 ; otherwise, 0 .

$\operatorname{De}(i, k p)$ : If instrument of type $k$ is used in period $p$ by surgery $i$, it takes 1 ; otherwise, 0 .

$\operatorname{IP}(k, p)$ : The inventory level of instrument $k$ in period $p$.

$\operatorname{Omax}(r)$ : The finish time of the last surgery of each operating room.

$\operatorname{Teta}(r, p)$ : The occupation status of room $r$ in block $p$. If room $r$ is occupied, it takes 1 ; otherwise, 0 .

Two $(r)$ : Idle time for each operating room.

\subsection{The Mathematical Model}

This model aims at minimizing operating room idle time and Cmax, where Cmax is, as mentioned earlier, the completion time of the latest patient's surgery in operating room. The most important objective of decision maker is minimizing the operating room idle time. But considering this objective alone in the model makes some operating rooms remain empty while there may be some operating rooms which are occupied up to a maximum possible. Therefore, minimizing $C \max$ is considered as the primary objective, and minimizing 
operating room idle time is the second level objective. Here, $f 1$ is defined Cmax and $f 2$ is the total idle time of operating rooms. Problem-solving process will be explained in a next section.

The MIP model is then formulated as follows:

$$
\begin{gathered}
f 1=\text { CMAX, } \\
f 2=\sum_{r} \operatorname{TWO}(r) .
\end{gathered}
$$

The constraints are

$$
\begin{aligned}
& \sum_{r} \sum_{s} \sum_{p} X_{i p s r}=1 \quad \forall i \\
& Y_{i p s r}=\sum_{p^{\prime}=\max (p-d(i)+1,1)}^{p} X_{i p^{\prime} s r} \quad \forall i, \forall p, \forall s, \forall r, \\
& \sum_{i} \sum_{s} Y_{i p s r} \leq 1 \quad \forall p, \forall r, \\
& \sum_{i} \sum_{r} Y_{i p s r} \leq 1 \quad \forall s, \forall p, \\
& \sum_{p} \sum_{r} X_{i p s r} \leq \mathrm{FS}(i, s) \quad \forall i, \forall s, \\
& \sum_{r} \sum_{i} Y_{i p s r} \leq \operatorname{SAV}(s, p) \quad \forall s, \forall p ， \\
& \sum_{p} \sum_{s} A_{i s p}=1 \quad \forall i: \operatorname{IsNA}(i)=1, \\
& \sum_{i: \operatorname{IsNA}(i)=1} \mathrm{AP}_{i s p}+\sum_{i} \sum_{r} Y_{i p s r} \leq 1 \quad \forall p, \forall s, \\
& \sum_{p} A_{i s p} \leq \operatorname{FAS}(i, s) \quad \forall i: \operatorname{IsNA}(i)=1, \forall s, \\
& \sum_{r} \sum_{s} X_{i p s r}=\sum_{s^{\prime} \neq s} A_{i s^{\prime} p} \quad \forall p, \forall i: \operatorname{IsNA}(i)=1, \\
& \mathrm{AP}_{\text {isp }}=\sum_{p^{\prime}=\max (p-d(i)+1,1)}^{p} A_{i s^{\prime} p} \quad \forall i: \operatorname{IsNA}(i)=1, \forall s^{\prime}, \forall p, \\
& \sum_{s^{\prime}} \sum_{r} Y_{i p s^{\prime} r} * \operatorname{AtN}(i, s) \leq \operatorname{SAV}(s, p) \quad \forall s: \operatorname{SGC}(s)=3, \forall s^{\prime}: \operatorname{SGC}\left(s^{\prime}\right)=1,2, \forall p, \forall i, \\
& \sum_{s^{\prime}} \sum_{r} Y_{i p s^{\prime} r} * \operatorname{Nat}(i) \leq \operatorname{Noat}(p) \quad \forall i, \forall p, \forall s^{\prime}: \operatorname{SGC}\left(s^{\prime}\right)=1,2
\end{aligned}
$$




$$
\begin{aligned}
& \sum_{i} \sum_{p} \sum_{r} Y_{i p s r}-\operatorname{mean}(l) \geq-\operatorname{dev}(l) \quad \forall l, \forall s: \mathrm{GN}(s, l)=1 \text {, } \\
& \sum_{p} \sum_{s} X_{i p s r} \leq \operatorname{FR}(i, r) \quad \forall i, \forall r \\
& \sum_{r} \sum_{s} \sum_{i} Y_{i p s r} \leq \operatorname{TecNo}(p) \quad \forall p, \\
& \operatorname{De}(i, k, p)=\sum_{r} \sum_{s} \operatorname{ins}(i, k) * X_{i p s r} \quad \forall i, \forall k, \forall p, \\
& \sum_{i} \operatorname{De}(i, k, p-d(i)-T S(k))+\operatorname{IP}(k, p-1)=\operatorname{IP}(k, p)+\sum_{i} \operatorname{De}(i, k, p), \\
& \operatorname{IP}(k, 0)=\operatorname{IP0}(k) \quad \forall k, \\
& \sum_{s} \sum_{i^{\prime} \text { :infect }\left(i^{\prime}\right)=0} X_{i^{\prime} p^{\prime} s r} \leq 1-\sum_{s} \sum_{i: \text { infect }(i)=1} X_{i p s r} \quad \forall p, \forall p^{\prime}: p \leq p^{\prime} \leq p+d(i)+1+\text { Kclean, } \\
& \operatorname{OMax}(r) \geq \sum_{i} \sum_{s} p * Y_{i p s r} \quad \forall r, \forall p, \\
& \operatorname{Teta}(r, p)=\sum_{i} \sum_{s} Y_{i p s r} \quad \forall r, \forall p, \\
& \operatorname{TWO}(r)=\operatorname{OMax}(r)-\sum_{p} \operatorname{Teta}(r, p) \quad \forall r, \\
& \sum_{p} Y_{i p s r} * p=d(i) * \sum_{p} X_{i p s r} \quad \forall i, \forall s, \forall r, \\
& \text { CMAX }-\sum_{s} \sum_{r} p * Y_{i p s r} \geq 0 \quad \forall i, \forall p, \\
& C M A X \leq \mathrm{C} 0 \text {, } \\
& \sum_{p} \sum_{s} \sum_{r} p * X_{i p s r} \leq \text { P0 } \quad \forall i: \operatorname{priority}(i)=1,
\end{aligned}
$$

where

$$
\begin{gathered}
X_{i p s r} \in\{o, 1\}, \quad A_{i s p} \in\{o, 1\}, \quad Y_{i p s r} \in\{o, 1\}, \\
\operatorname{AP}_{i s p} \in\{o, 1\}, \quad \operatorname{Teta}(r, p) \in\{o, 1\}, \quad \operatorname{De}(i, k, p) \in\{o, 1\}, \\
\operatorname{TWO}(r)>0, \quad \operatorname{OMax}(r)>0, \quad \operatorname{IP}>0 .
\end{gathered}
$$

Equations (4.1) and (4.2) define $f 1$ (Cmax) and $f 2$ (total idle time of operating rooms), respectively. Equation (4.3) states that each surgery should be assigned to just one operating room, one surgeon, and one time block as a start block. The variable $Y_{i p s r}$ is defined in (4.4). At most, one surgery can be operated in each operating room in each time block (see (4.5)). Equation (4.6) states that the number of simultaneous operations performed by 
each surgeon cannot exceed one. Equation (4.7) specifies that the operation should not be assigned to the surgeon that is not qualified for it. Constraint (4.8) prohibits assigning a surgical case to a surgeon who is not available in that period. Constraints (4.9) to (4.13) relate to assigning assistant surgeons to operations. Constraints (4.14) and (4.15) are related to the availability of attending surgeons during the operations. Equation (4.16) defines the balanced distribution of operations between several surgeon groups. Equation (4.17) forbids an operation in a room where the required equipment is not available. Constraint (4.18) ensures that the required technicians for all the surgical cases performed simultaneously cannot exceed the total number of technicians available in that period. Constraints (4.19) to (4.21) state that the number of required instruments cannot exceed the available capacity. We consider medical precaution constraints for infected patients also considered by [16]. Constraint (4.22) states that, when the surgery of an infected patient is scheduled for a specific period in an OR, the noninfected patient cannot be assigned to that OR until additional cleaning are performed. Constraint (4.23) determines the finish time of the last surgery of each operating room. The occupation of $r$ th room in $p$ th time block is defined by the variable $\operatorname{Teta}(r, p)$ in (4.24). Constraint (4.25) is used to define the total idle time (TWO) for each operating room. Equation (4.26) states that no surgery can start too late but must be planned to be completed within the operation room opening hours. Equations (4.27) and (4.28) ensure that end time of last operation should not exceed form allowable time and, finally, (4.29) defines priority constraint.

\section{Solving Approach}

As previously mentioned, this model aims at minimizing operating room idle time and Cmax. Initially the problem was solved using the Lexicograph method. Due to the high time to solve, some changes were made in solving method. Below, $f 1$ and $f 2$ are $C \max$ and idle time of operating rooms, respectively, and $G_{i}(x)$ shows constraints of the model. Solving process is described below.

Step 1. Choose an appropriate upper bound for $f 1$.

Step 2. Solve the following problem:

$$
\begin{gathered}
\operatorname{Min} \quad f 2(X) \\
\text { s.t. : } \quad G_{i}(X) \leq 0 ; i=1, \ldots, M \\
f 1(x) \leq \text { Upper bound. }
\end{gathered}
$$

Step 3. If the problem is feasible, deduce the upper bound and go to Step 2.

Step 4. If the problem has no feasible solution, then exit with the current feasible solution as the optimum solution.

Flow diagram of this method is shown in Figure 1. 


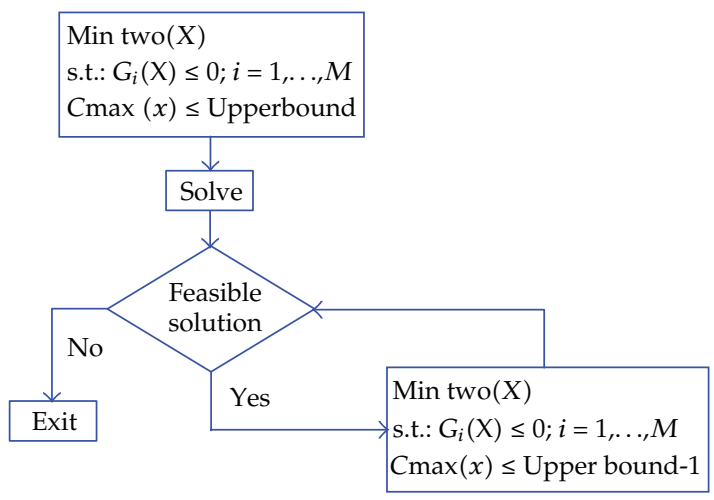

Figure 1: Flow diagram of the solving method.

\section{Computational Experiments}

We did not come across any problem as defined in this paper. Although there are different works cited in the literature, the differences exist in the constraints considered. In this paper, the real constraints of teaching hospitals have been duly taken into account.

To evaluate the proposed mathematical model, several real problems were collected from the Hasheminejad Kidney Center (HKC), an academic hospital, in Iran. The collected data consisted of lists of patients for each day, start and end times of surgeries, details of the patients such as infection, the surgeon assigned, and the operating room assigned. To collect some required data that is not registered in the HIS (hospital information system), we have designed an ICR (intelligent character recognition) form that was completed by operating room personnel for 6 months and selected the most complete forms that were filled out within a one-month period. The designed form is shown in the appendix section.

There are four different surgical services at HKC: urologic, endoscopic, laparoscopic, and vascular. The operating theatre is composed of 7 operating rooms. All the operating rooms are multifunctional, but endoscopic operations can be performed in only three. There are four resident groups of urology according to their seniority levels and two fellowship groups. Each resident group consists of three residents with the same skill, and each fellowship group consists of two fellows. There is a trainee rotation that determines the days each resident or fellow should serve in the operating theatre.

The number of technician groups varies on different work shifts. There are 35 different types of important instruments used in the operating theatre (e.g., laparoscopic trolley, C-Arm, etc.), each case requiring a specific set. Furthermore, surgery durations, required instruments for each surgery, instrument inventory, and the corresponding sterilization times are known. Also the duration of setup time is non-sequence-dependent and is considered in the processing time of the operation.

Time data are expressed in 30-minute units, and the scheduling horizon comprises 25 time units.

The model was implemented in GAMS and solved using CPLEX 12.1.0. The allowed computation time for the solution procedure was limited to 400 seconds per instance on a 2, $66 \mathrm{GHz}$ Pentium 4 PC with the Windows 7 operating system.

The model was evaluated by solving several real instances at $\mathrm{HKC}$, and the gap from optimal solution was reported. 
Table 1: Results of 30 test instances.

\begin{tabular}{|c|c|c|c|c|c|c|c|}
\hline $\begin{array}{l}\text { Sample } \\
\text { number }\end{array}$ & patients & Surgeons & $\begin{array}{c}\text { Discrete } \\
\text { var. }\end{array}$ & Constraints & $\begin{array}{c}\text { MIP } \\
\text { absolute gap }\end{array}$ & $\begin{array}{c}\text { Best } \\
\text { solution }\end{array}$ & $\begin{array}{c}\text { CPU time to } \\
\text { optimal }\end{array}$ \\
\hline Instance 1 & 18 & 7 & 91,675 & 58,397 & 0 & 0 & 15.000 \\
\hline Instance 2 & 18 & 9 & 123,225 & 75,300 & 0 & 0 & 190.531 \\
\hline Instance 3 & 23 & 8 & 157,250 & 95,216 & 3 & 0 & 1201.281 \\
\hline Instance 4 & 24 & 9 & 164,675 & 95,590 & 0 & 0 & 316.844 \\
\hline Instance 5 & 24 & 8 & 164,175 & 99,437 & 0 & 0 & 346.953 \\
\hline Instance 6 & 25 & 6 & 136,000 & 84,842 & 0 & 0 & 259.351 \\
\hline Instance 7 & 25 & 12 & 162,200 & 98,783 & 5 & 3 & 1534.626 \\
\hline Instance 8 & 26 & 10 & 160,825 & 99,105 & 0 & 0 & 23.610 \\
\hline Instance 9 & 26 & 9 & 165,600 & 101,407 & 0 & 0 & 100.907 \\
\hline Instance 10 & 27 & 12 & 184,750 & 158,036 & 0 & 0 & 81.562 \\
\hline Instance 11 & 27 & 10 & 166,950 & 102,872 & 0 & 0 & 83.078 \\
\hline Instance 12 & 27 & 10 & 157,550 & 97,340 & 0 & 0 & 113.359 \\
\hline Instance 13 & 28 & 12 & 182,975 & 111.671 & 0 & 0 & 69.534 \\
\hline Instance 14 & 28 & 10 & 183,075 & 111,788 & 3 & 0 & 1415.297 \\
\hline Instance 15 & 29 & 10 & 177,175 & 108,016 & 1 & 0 & 1601.735 \\
\hline Instance 16 & 31 & 9 & 179,250 & 109,769 & 0 & 0 & 27.578 \\
\hline Instance 17 & 32 & 14 & 275,275 & 161,770 & 0 & 0 & 46.078 \\
\hline Instance 18 & 32 & 13 & 220.275 & 133,186 & 0 & 0 & 255.468 \\
\hline Instance 19 & 32 & 9 & 197,825 & 121,318 & 0 & 0 & 370.190 \\
\hline Instance 20 & 32 & 8 & 183,025 & 111,299 & 3 & 0 & 1621.750 \\
\hline Instance 21 & 33 & 10 & 214,000 & 129,670 & 0 & 0 & 41.313 \\
\hline Instance 22 & 33 & 11 & 231,840 & 142,729 & 0 & 0 & 342.719 \\
\hline Instance 23 & 34 & 13 & 268,625 & 158,940 & 0 & 0 & 39.157 \\
\hline Instance 24 & 34 & 9 & 161,025 & 101,346 & 2 & 0 & 908.891 \\
\hline Instance 25 & 35 & 13 & 326,175 & 184,021 & 0 & 0 & 73.875 \\
\hline Instance 26 & 36 & 8 & 222,975 & 136,670 & 0 & 0 & 44.015 \\
\hline Instance 27 & 36 & 10 & 210,475 & 130,185 & 0 & 0 & 46.968 \\
\hline Instance 28 & 37 & 9 & 226,900 & 138,236 & 1 & 0 & 430.109 \\
\hline Instance 29 & 39 & 10 & 240,075 & 139,643 & 0 & 0 & 50.328 \\
\hline Instance 30 & 45 & 10 & 293,900 & 178,170 & 4 & 0 & 1338.461 \\
\hline
\end{tabular}

The evaluation was based on 30 real test problems with 18 to 45 patients and 8 to 13 surgeons. To study the performance of the model, the MIP gap was evaluated. Table 1 shows the resulting MIP gap for each test instance defined as the absolute gap between the values obtained for the objective function and the lower bound given by CPLEX, the number of discrete variables, constraints, patients, and surgeons.

In addition, to analyze the surgical case scheduling resulting from the proposed model, the final solution for one month of chosen instances was explored and the actual scheduling of hospital compared with the proposed scheduling.

Since number and type of surgery varies in different days of a week, 7 patterns to choose samples in different days were considered. Training operations are not performed on Thursday and Friday. Also transplant operations with their specific constraints are performed 


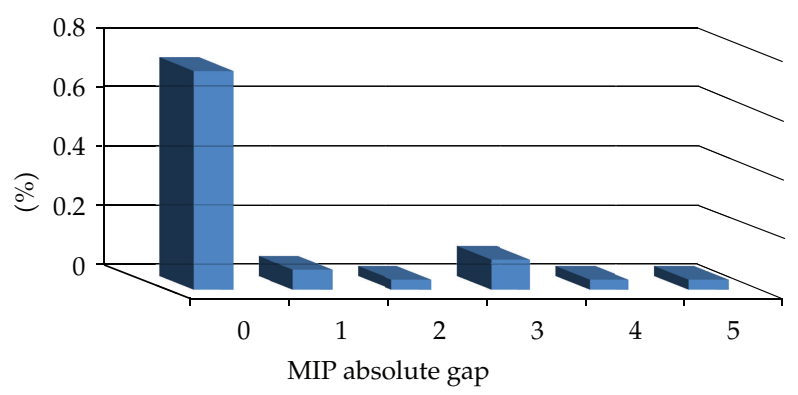

Figure 2: Frequency of the results in the range for 30 real instances within the specified computation time.

Table 2: Evaluation result of proposed model (HORS).

\begin{tabular}{|c|c|c|c|c|c|}
\hline \multirow{2}{*}{ Sample issue } & \multicolumn{2}{|c|}{$C \max$} & \multicolumn{2}{|c|}{ Idle time } & \multirow{2}{*}{ Elapsed time (s) } \\
\hline & HORS & HKC & HORS & HKC & \\
\hline 1 & $16: 30$ & $18: 30$ & 0 & 450 & 54.000 \\
\hline 2 & $16: 00$ & $16: 30$ & 30 & 330 & 88.672 \\
\hline 3 & $15: 00$ & $16: 45$ & 0 & 255 & 69.391 \\
\hline 4 & $16: 30$ & $19: 30$ & 0 & 292.5 & 64.125 \\
\hline 5 & $20: 30$ & $21: 30$ & 0 & 315 & 307.000 \\
\hline 6 & $15: 30$ & $20: 00$ & 0 & 480 & 61.515 \\
\hline 7 & $17: 30$ & 19:15 & 240 & 337.5 & 215.719 \\
\hline 8 & 15:00 & $18: 30$ & 0 & 292.5 & 68.359 \\
\hline 9 & $15: 30$ & $18: 15$ & 0 & 283.5 & 73.454 \\
\hline 10 & 14:30 & $17: 30$ & 0 & 352.5 & 32.563 \\
\hline 11 & $14: 30$ & $17: 30$ & 0 & 217.5 & 32.031 \\
\hline 12 & 19:30 & $19: 20$ & 0 & 270 & 124.843 \\
\hline
\end{tabular}

on Sunday and Tuesday. Thereby the problem instances are not chosen from these days of the week (because in this model, focus is on training operations and constraints of transplant operations are not considered).

Because of the complexities of this problem, no predictive scheduling considering all surgeries and all constraints can be done by the OR head nurse or manager of the operating theatre. So the comparison of the obtained schedule from HORS and the predictive schedule of the hospital is not possible. In HKC, after completing each surgery, the OR head nurse schedules the next case considering the actual system status at that moment. Hence, an online evaluation is proposed that includes the actual system status. In this validation method, the proposed model is updated each hour periodically. In addition the rescheduling is done after the end of each operation or emergency patient entry. Thereby, the Hybrid Rescheduling Policy is used.

The gap evaluations for the real instances show that the results are in general satisfactory. The smallest and greatest absolute gaps were 0 and 5 in the allowed computation time and $73 \%$ of tested cases reached optimal solutions in the specified time; even the time needed to find optimal solutions for the all of the test instances was lower than $1700 \mathrm{~s}$ (about $30 \mathrm{~min}$ ) and could, hence, be still considered as reasonable. Figure 2 shows the frequency of the results in the range, verifying the efficiency of the proposed approach. 
Table 3: Average results of 12 samples examined.

\begin{tabular}{lcc}
\hline Average results & HORS & HKC \\
\hline Average operating room idle time (min/day) & 22.5 & 323 \\
Average Cmax & $16: 22$ & $18: 35$ \\
\hline
\end{tabular}

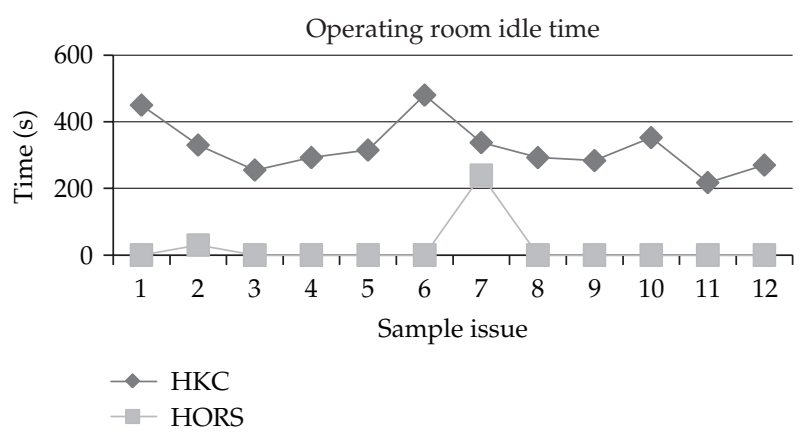

Figure 3: Comparison of "operating room idle time" between Hasheminejad scheduling (HKC) and proposed scheduling (HORS).

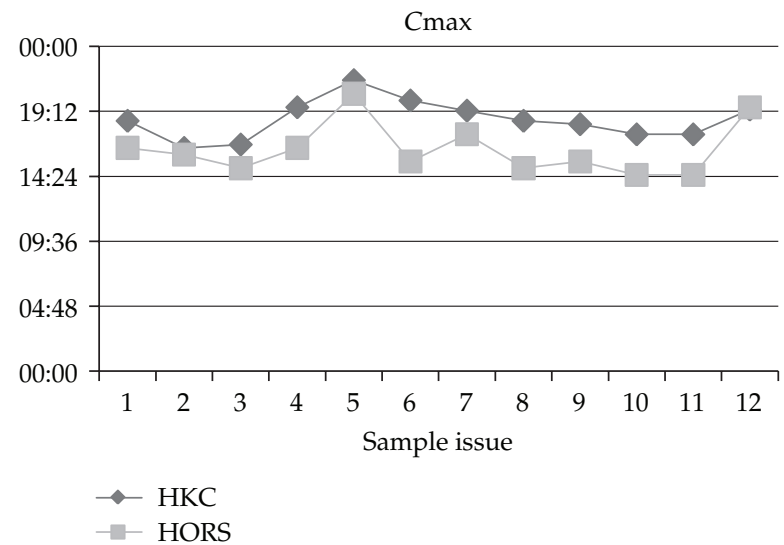

Figure 4: Comparison of "Cmax" between Hasheminejad scheduling (HKC) and proposed scheduling (HORS).

The result of comparison between actual scheduling and proposed model (HORS) based on the value of operating room idle time and Cmax are presented in Table 2.

From the results presented in Table 2, the solutions provided by "HORS" scheduling are better than those of the HKC scheduling. Table 3 shows the mean of obtained result.

Averagely, idle time operating room in HKC was 323 minutes per day and Cmax was 18:30 while these results for proposed model, respectively, were 22.5 minutes per day and 16:22.

Figures 3 and 4 show comparison of "operating room idle time" and Cmax between the Hasheminejad scheduling (HKC) and the proposed model (HORS), respectively. 


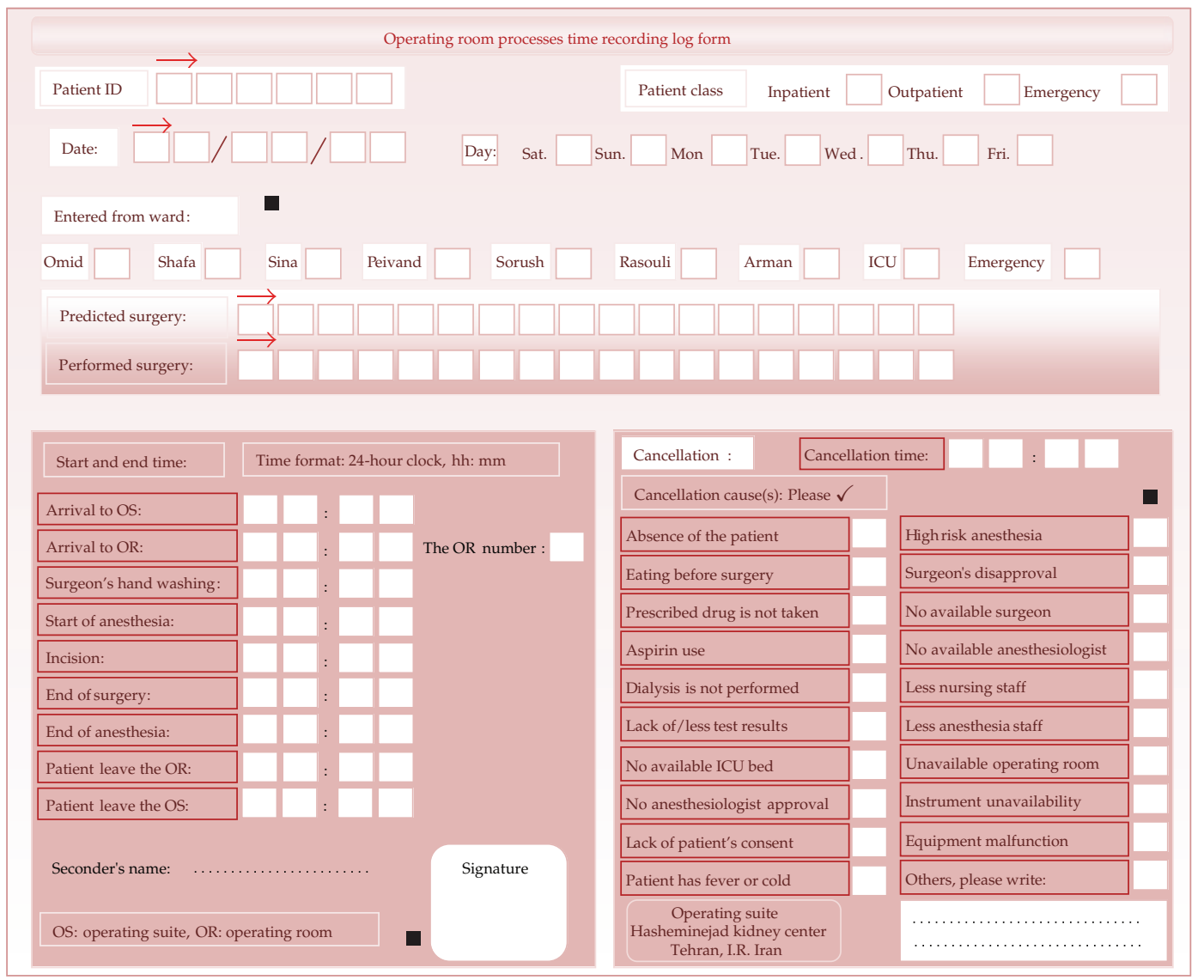

Figure 5: Operating room processes time recording log form.

\section{Conclusions and Recommendations}

In this paper, the second step of the surgical process scheduling was investigated for scheduling elective patients waiting surgery. We formulated a mixed integer problem to assign a set of operations to some resources consisting of human resources (surgeon, assistant surgeon) and applied resource (OR) and sequenced them simultaneously. Taking into account the training plan of residents and fellows, real constraints in teaching hospitals and assignment of assistant surgeons to each operation form the main features of the proposed model.

The proposed method was evaluated and verified through solving several real instances at a teaching hospital. Also, the final solution for one month of chosen instances was explored and the actual scheduling of hospital compared with the proposed scheduling.

Numerical results indicated the efficiency of the proposed model compared to the actual hospital scheduling, and the gap evaluations for the real instances showed that the results were generally satisfactory.

The proposed model can be extended by including other resources such as ICU bed or ward bed in this model. We are working in a deterministic context; for future work, we will consider uncertainty related to surgery time and emergency patient arrival. Another 
suggestion for future work is to use the constraint programming, as a viable and powerful method for modeling and solving many combinatorial problems, to solve the described problem.

\section{Appendix}

\section{The Designed ICR (Intelligent Character Recognition) Form}

For more details, see Figure 5.

\section{Acknowledgments}

This research was conducted in part using data and resources from the Hasheminejad Kidney Center (HKC), a tertiary hospital of the Tehran University of Medical Sciences (TUMS). The authors are grateful to the contribution of Dr. Etemadian in providing access to key research materials. The contributions of HKC's operating rooms staff are gratefully acknowledged.

\section{References}

[1] J. Haynes, UK Centre for the Measurement of Government Activity Expenditure on Healthcare in the UK, The Office of Public Sector Information, Norwich, UK, 2010.

[2] S. Chaabane, N. Meskens, A. Guinet, and M. Laurent, "Comparison of two methods of operating theatre planning: application in Belgian hospital," in Proceedings of the International Conference on Service Systems and Service Management (ICSSSM '06), Troyes, France, October 2006.

[3] J. M. Magerlein and J. B. Martin, "Surgical demand scheduling: a review," Health Services Research, vol. 13, no. 4, pp. 418-433, 1978.

[4] D. N. Pham and A. Klinkert, "Surgical case scheduling as a generalized job shop scheduling problem," European Journal of Operational Research, vol. 185, no. 3, pp. 1011-1025, 2008.

[5] B. Roland, C. D. Martinelly, F. Riane, and Y. Pochet, "Scheduling an operating theatre under human resource constraints," Computers and Industrial Engineering, vol. 58, no. 2, pp. 212-220, 2010.

[6] J. T. Blake and M. W. Carter, "Surgical process scheduling: a structured review," Journal of the Society for Health Systems, vol. 5, no. 3, pp. 17-30, 1997.

[7] B. Cardoen, E. Demeulemeester, and J. Belien, “Operating room planning and scheduling: a literature review," European Journal of Operational Research, vol. 201, no. 3, pp. 921-932, 2010.

[8] M. Kennedy, Bin-packing, knapsack and chance constrained approaches to scheduling operating rooms, Ph.D. thesis, Rensselaer Polytechnic Institute, Troy, NY, USA, 1992.

[9] S. N. Ogulata and R. Erol, "A hierarchical multiple criteria mathematical programming approach for scheduling general surgery operations in large hospitals," Journal of Medical Systems, vol. 27, no. 3, pp. 259-270, 2003.

[10] J. Vissers, I. Adan, and J. A. Bekkers, "Patient mix optimization in tactical cardiothoracic surgery planning: a case study," IMA Journal of Management Mathematics, vol. 16, no. 3, pp. 281-304, 2005.

[11] A. Jebali, A. B. Hadj Alouane, and P. Ladet, "Operating rooms scheduling," International Journal of Production Economics, vol. 99, no. 1-2, pp. 52-62, 2006.

[12] A. Testi, E. Tanfani, and G. Torre, "A three-phase approach for operating theatre schedules," Health Care Management Science, vol. 10, no. 2, pp. 163-172, 2007.

[13] H. Fei, C. Chu, N. Meskens, and A. Artiba, "Solving surgical cases assignment problem by a branchand-price approach," International Journal of Production Economics, vol. 112, no. 1, pp. 96-108, 2008.

[14] A. Riis and E. Burke, "Surgery allocation and scheduling," in Proceedings of the 7th International Conference on the Practice and Theory of Automated Timetabling, Montreal, Canada, 2008.

[15] B. Cardoen, E. Demeulemeester, and J. Beliën, "Sequencing surgical cases in a day-care environment: an exact branch-and-price approach," Computers and Operations Research, vol. 36, no. 9, pp. 2660-2669, 2009. 
[16] H. Fei, C. Chu, N. Meskens, and A. Artiba, "A planning and scheduling problem for an operating theatre using an open scheduling strategy," Computers and Industrial Engineering, vol. 58, no. 2, pp. 221-230, 2010.

[17] A. Hanset, N. Meskens, and D. Duvivier, "Using constraint programming to schedule an operating theatre," in Proceedings of the IEEE Workshop on Health Care Management (WHCM '10), pp. 1-6, February 2010.

[18] A. Hanset, N. Mesken, and D. Duvivier, "Comparison of two models to schedule the operating theatre," in Proceedings of the 8th International Conference of Modeling and Simulation (MOSIM '10), Hammamet, Tunisia, May 2010.

[19] S. Topaloglu and I. Ozkarahan, "A constraint programming-based solution approach for medical resident scheduling problems," Computers \& Operations Research, vol. 38, no. 1, pp. 246-255, 2011.

[20] H. D. Sherali, M. H. Ramahi, and J. Saifeeq, "Hospital resident scheduling problem," Production Planning and Control, vol. 13, no. 2, pp. 220-233, 2002.

[21] F. Dexter, R. E. Wachtel, R. H. Epstein, J. Ledolter, and M. M. Todd, "Analysis of operating room allocations to optimize scheduling of specialty rotations for anesthesia trainees," Anesthesia and Analgesia, vol. 111, no. 2, pp. 520-524, 2010. 


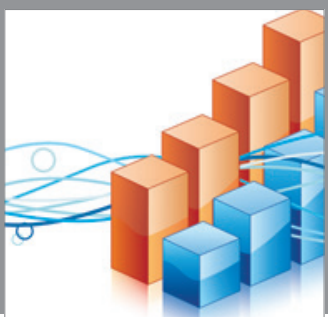

Advances in

Operations Research

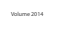

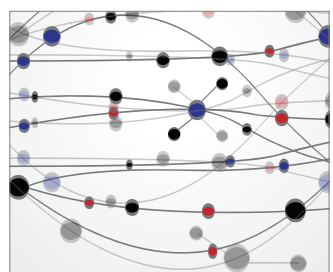

\section{The Scientific} World Journal
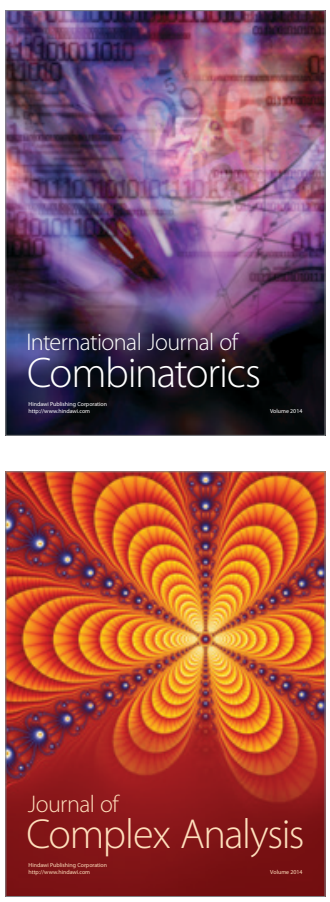

International Journal of

Mathematics and

Mathematical

Sciences
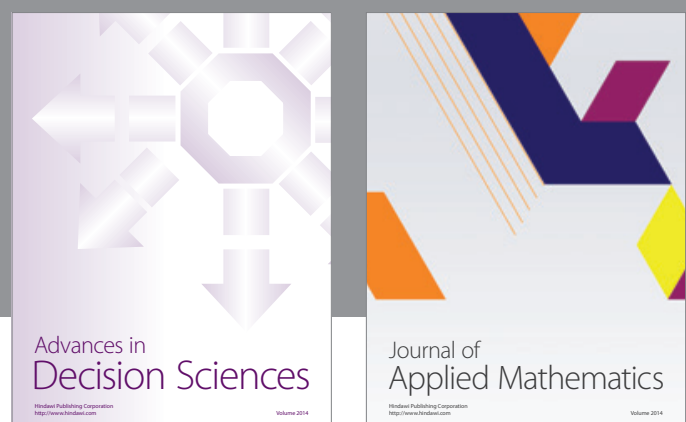

Journal of

Applied Mathematics
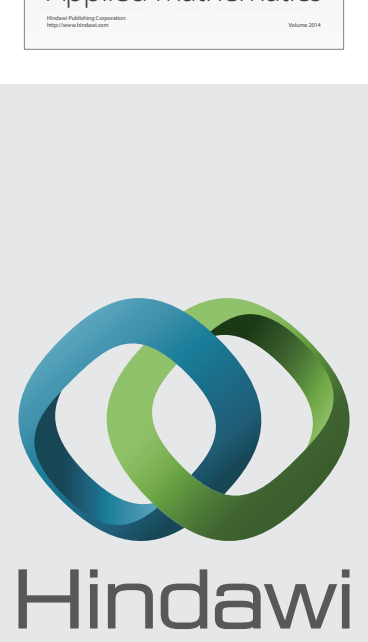

Submit your manuscripts at http://www.hindawi.com
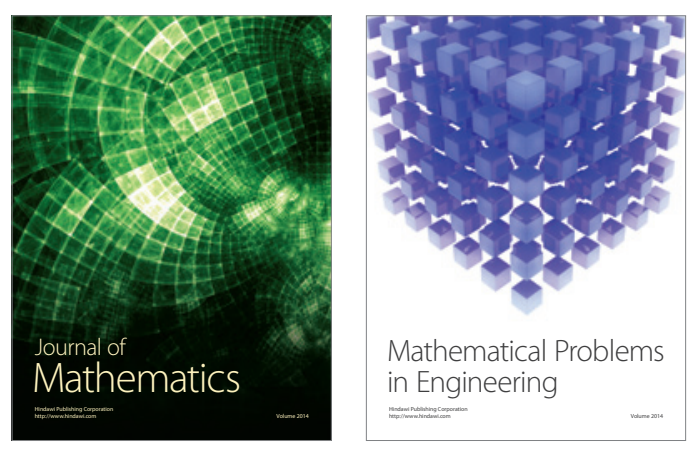

Mathematical Problems in Engineering
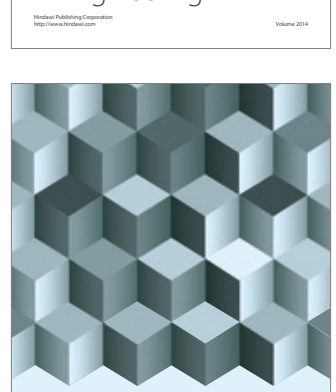

Journal of

Function Spaces
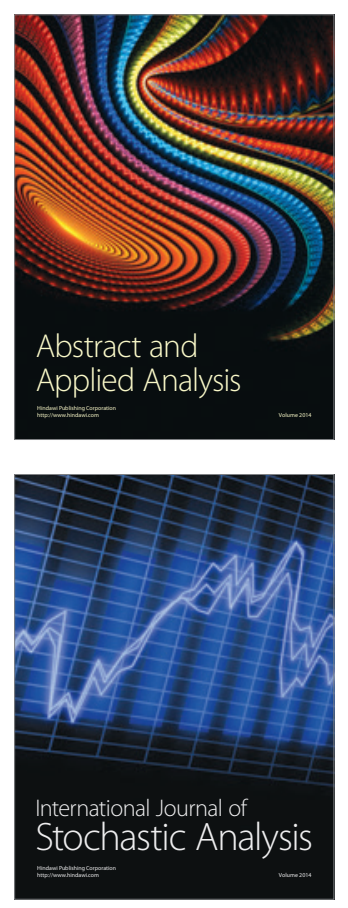

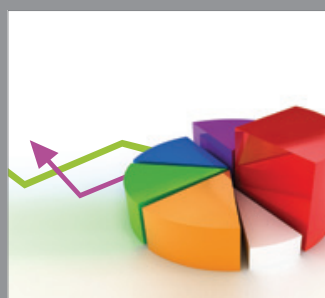

ournal of

Probability and Statistics

Promensencen
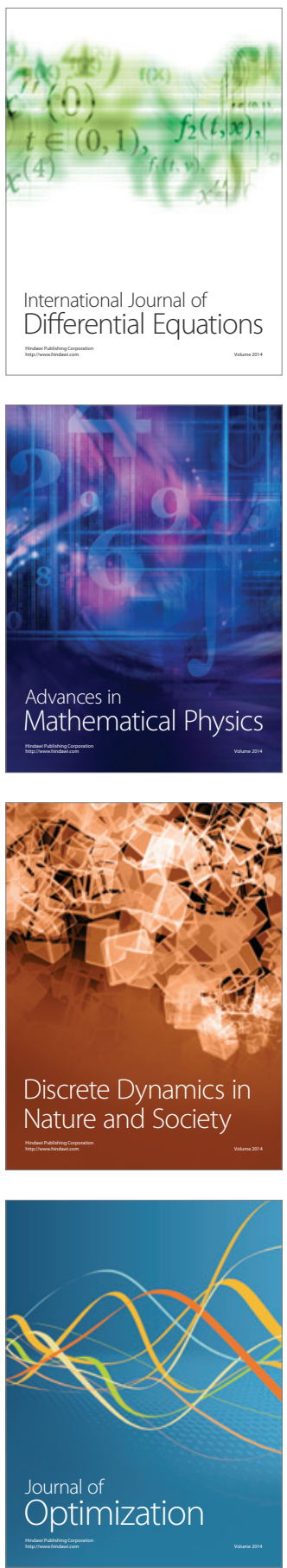